lacked TLR expression. In vitro, TLR9 stimulation induced proliferation of transitional $B$ cells, activation and maturation as MZ B cells (Notch2high, CD21high, IgMhigh, IgDlow, CD23low, CD27). Mature B cells also proliferated but differentiated toward a follicular pathway (Notch2low, IgMlow, IgDlow, CD23low, CD27). Production of Ig was observed in both B cell supernatants.

Conclusion High TLR level in transitional salivary gland B cell aggregates indicates how highly sensitive to inflammatory stimuli they are. Immature B cells can differentiate into Ig-secreting cells following TLR9 stimulation through a MZ-like maturation pathway without entering the germinal centres. In contrast, mature B cells differentiate towards a classical follicular pathway. Whether one or both differentiation pathways generate autoreactive B cells in salivary glands remains to be established in patients with Sjögren's syndrome.

\section{A105 TOLL-LIKE RECEPTOR 9 (TLR9) CONTROLS B CELL DIFFERENTIATION IN SALIVARY GLANDS OF PATIENTS WITH SJÖGREN'S SYNDROME}

C Jamin, T Guerrier, L Le Pottier, A Saraux, P Youinou, J Pers EA 2216 « Immunologie et Pathologie " and IFR 148 ScInBioS, Université de Brest Université Européenne de Bretagne, Brest, France

\subsection{6/ard.2010.129627r}

Background B cell clusters represent genuine germinal centres in the salivary glands of patients with Sjögren's syndrome, but the majority display transitional and marginal zone (MZ)-like $B$ cell characteristics. The authors questioned whether TLR9 stimulation, which occurs during inflammatory reactions, could influence such an aberrant B cell differentiation.

Methods Following laser microdissection, TLR expression was measured in B cell aggregates using quantitative RT-PCR. As controls, transitional and mature B cells from cord blood and tonsils were FACS sorted and stimulated with CpG-ODN. CFSE enabled evaluation of their proliferation, and they were also analysed by flow cytometry to determine their activation status. Differentiation into Ig-secreting cells was demonstrated by ELISA.

Results Transitional B cell aggregates of salivary glands expressed a high level of TLRs whereas real germinal centres 